\title{
“Hidden” QTc prolongation induced by olanzapine.
}

Hirofumi Hirakawa ${ }^{1}$, M.D., Takeshi Terao ${ }^{1}$, M.D., Ph.D., Naohiko Takahashi ${ }^{2}$, M.D., Ph.D.

1) Department of Neuropsychiatry, Oita University Faculty of Medicine

2) Department of Cardiology and Clinical Examination, Oita University Faculty of Medicine

$<$ Background $>$

Prolongation of the corrected QT interval (QTC) is an important finding because it increases the risk of torsade de pointes, which can be fatal and cause sudden cardiac death. ${ }^{1}$ A normal QTC interval is generally defined as 360 to $450 \mathrm{~ms}$ for male and 370 to $460 \mathrm{~ms}$ for female. ${ }^{2}$ QTc longer than $500 \mathrm{~ms}$ is considered prolonged and confers increased risk of torsade de pointes. ${ }^{1}$ Olanzapine, one of the most widely used antipsychotics, is known to increase the risk of QTc prolongation.3,4

\section{$<$ Objective>}

To alert clinicians to the risk of QTc prolongation induced by olanzapine that is only occurred after exercise and was not apparent in the resting electrocardiography (ECG).

\section{$<$ Case presentation>}

A 45-year-old Japanese woman with schizophrenia treated with $6 \mathrm{mg}$ per day of aripiprazole was attending our hospital. She and her family had no history of cardiovascular disease. At initial presentation to the hospital prior to antipsychotic treatment, ECG found that the QTc was 417 ms. On April 2017, her auditory hallucinations were exacerbated and $5 \mathrm{mg}$ per day of olanzapine was initiated, while aripiprazole was decreased to $3 \mathrm{mg}$ per day. After starting olanzapine therapy, her auditory hallucinations improved. However, she sometimes felt palpitations and was seen by the cardiology department in our hospital in September 2018. Her ECG showed no abnormalities and the QTc was 434 ms. Her echocardiography showed no apparent structural abnormalities. Treadmill exercise stress testing was performed in October 2018. During and after exercise, no ischemic ST changes was seen. The QTc increased from $400 \mathrm{~ms}$ before the exercise test to $540 \mathrm{~ms}$. We suspected the adverse effect of olanzapine because the risk of QTC prolongation was much higher than that of aripiprazole. ${ }^{3}$ Olanzapine was discontinued immediately, and aripiprazole was increased to $6 \mathrm{mg}$ per day. After discontinuation of olanzapine, the treadmill exercise test was repeated in December 2018. The QTC returned to the normal range, increasing from $370 \mathrm{~ms}$ before the exercise test to $420 \mathrm{~ms}$.

\section{$<$ Discussion $>$}

In this case, olanzapine-induced QTc prolongation only occurred after exercise and was not apparent in the resting ECG.
ECG

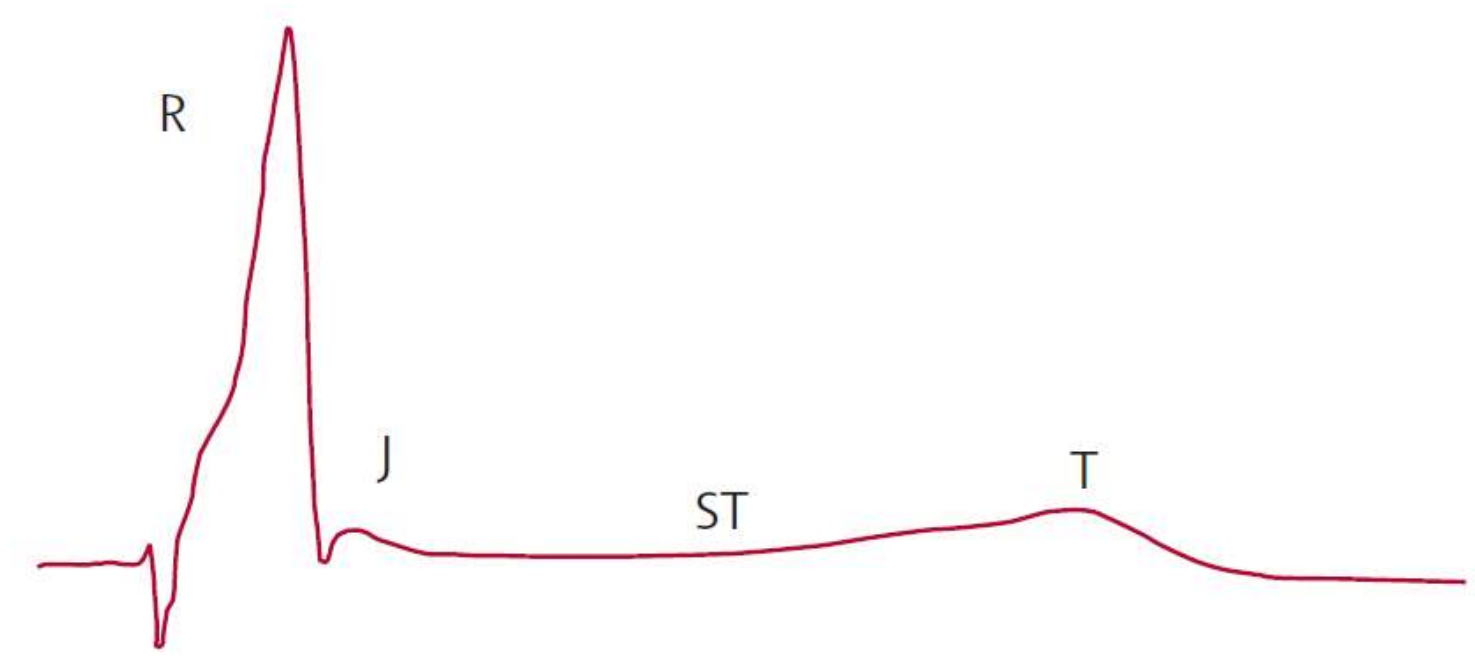

Action potential

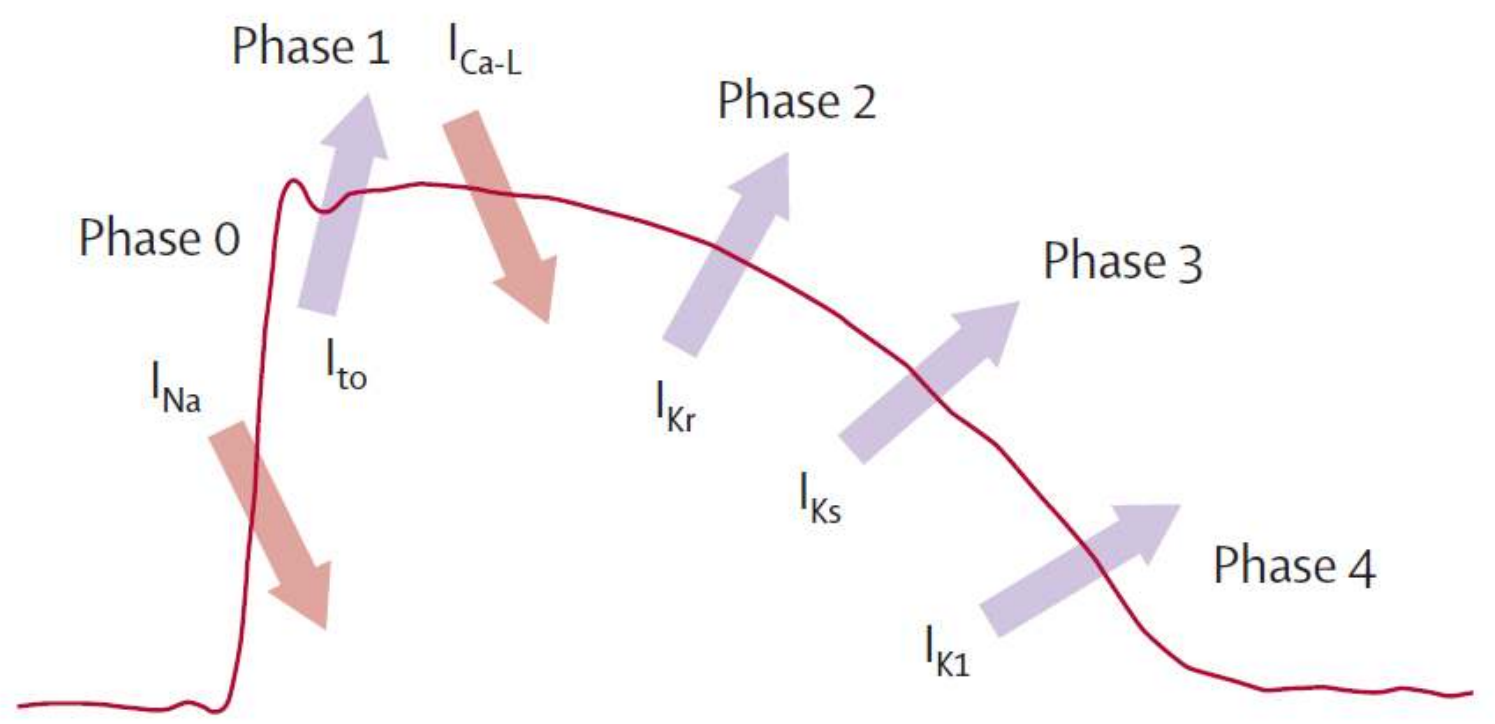

Figure. Ventricular action potential and major ion currents. ${ }^{1}$

The QT interval indicates the duration of ventricular depolarization and repolarization, that is caused by transmembrane flow of ions, especially an outward flow of potassium through two delayed rectifier potassium currents, rapid component (IKr) and slow component (IKs). In congenital long QT syndrome (LQTS), mutations in the genes encoding for potassium channels cause malfunction of IKr or IKs. ${ }^{1}$ Drug-induced LQTS, including induction by antipsychotics, is generally ascribed to blockade of the $\mathrm{IKr} .1,4$ In congenital LQTS, activities leading to sympathetic stimulation, such as exercise or emotional stress, trigger QTc prolongation. ${ }^{1} \mathrm{~A}$ previous cohort study found that users of atypical and typical antipsychotics had an increased risk of sudden cardiac death compared with nonusers. ${ }^{5}$ We suppose that a proportion of patients taking antipsychotics (including olanzapine) who died from sudden cardiac death might have such drug-induced QTC prolongation triggered by sympathetic stimulation during daily life although their routine ECG showed no abnormality. Although it is unclear whether only olanzapine or a combination of olanzapine with aripiprazole led to QTc prolongation in our patient, all clinicians should be cautious about such "hidden" QTc prolongation induced by olanzapine. If possible, it is desirable to perform exercise stress testing during olanzapine treatment. Further study is needed to confirm such antipsychotics-sympathetic stimulation induced QTc prolongation to prevent sudden cardiac death.

\section{$<$ References>}

1. Morita H, Wu J, Zipes DP. The QT syndromes: long and short. Lancet. 2008; 372, 750-763.

2. Viskin S. The QT interval: too long, too short or just right. Heart rhythm. 2009; 6: 711-715.

3. Leucht S, Cipriani A, Spineli L, et al. Comparative efficacy and tolerability of 15 antipsychotic drugs in schizophrenia: a multiple-treatments meta-analysis. Lancet. 2013; 382: 951-62.

4. Morissette $P$, Hreiche $R$, Mallet $L$, et al. Olanzapine prolongs cardiac repolarization by blocking the rapid component of the delayed rectifier potassium current. J Psychopharmacol. 2007; 21: 735- 41.

5. Ray WA, Chung CP, Murray KT , et al. Atypical antipsychotic drugs and the risk of sudden cardiac death. N Engl J Med. 2009; 360:225-35. 